\title{
Diagnostic experience of patients with fibromyalgia - a meta-ethnography
}

Anne Marit Mengshoel ${ }^{1}$

Julius Sim ${ }^{2}$

Birgitte Ahlsen ${ }^{1,3}$

Sue Madden ${ }^{4}$ a.m.mengshoel@medisin.uio.no

j.sim@keele.ac.uk

birgitte.ahlsen@medisin.uio.no

susan.madden@anglia.ac.uk

1 Institute of Health and Society, Medical Faculty, University of Oslo, Norway

2 Institute for Primary Care and Health Sciences, Keele University, United Kingdom

3 Faculty of Health Sciences, Oslo and Akershus University College of Applied Sciences, Norway

$4 \quad$ Faculty of Medical Science, Anglia Ruskin University, United Kingdom

\section{Corresponding author:}

Anne Marit Mengshoel

Department of Health Sciences, Institute of Health and Society, Medical Faculty, University of Oslo, Box 1153 Blindern, 0316 Oslo, Norway. e-mail: a.m.mengshoel@medisin.uio.no

Published in: Chronic Illness, 2017 doi: 10.1177/1742395317718035 [Epub ahead of print 1 Aug 2017]. 


\section{Abstract}

Objective: To examine how individuals experience the process and consequences of receiving a diagnosis of fibromyalgia syndrome (FMS).

Methods: A systematic literature search of qualitative studies up to May 2016 was performed. Twenty-eight reports including information on patients' diagnostic experiences were subjected to an interpretive analysis in accordance with the principles of meta-ethnography.

Results: Years were normally spent consulting specialists in an attempt to confirm the reality of symptoms and make sense of the illness. Great relief was felt at finally achieving the FMS diagnosis. However, relief waned when therapies proved ineffective. Health professionals and others questioned whether individuals were genuinely ill, that the illness had a psychological nature, and whether they were doing their best to recover. The diagnosis did not provide a meaningful explanation of individuals' suffering and had limited power to legitimate illness. Patients felt blamed for their failure to recover, threatening their personal credibility and moral identity.

Conclusion: The FMS diagnosis has limitations in validating and making sense of patients' illness experiences and in providing social legitimation of their illness. Social relationships are strained during the diagnostic process and in the course of ineffective therapies.

\section{Keywords}

Fibromyalgia - contested illness - diagnostic experience - legitimacy - metasynthesis - metaethnography 


\section{Introduction}

Diagnoses are applied in medicine to distinguish the ill from the healthy, and thereby determine who needs therapy and support and who does not. ${ }^{1}$ Accordingly, diagnoses mirror the medical understanding of disease, illness and health, which in turn shapes clinical practice. During the diagnostic process, physicians determine how to understand the patient's illness, as a basis for choosing appropriate clinical management; this in turn helps to shape the meaning of the illness experience for the patient. ${ }^{2}$ Hence, diagnostics includes both the process of reaching a diagnosis and the specific diagnostic category arrived at. ${ }^{3}$ Biomedical diagnoses reflect pre-specified criteria and are largely determined by defined patterns of objective signs of pathology (i.e. abnormal organ function), while mental and social diagnoses are defined by clusters of symptoms pointing respectively to mental problems (e.g. depression) or deviant behaviour (e.g. alcoholism). Unlike biomedical diagnoses, neither mental nor social diagnoses normally assert defined causes. ${ }^{4}$ However, distinctions between biomedical, mental and social diagnosis can be blurred.

The success of medicine is often portrayed through its objectivity and advanced technology, enabling doctors to detect physiological dysfunction. Through the diagnostic process, a patient's illness experiences become a named disease, i.e. an objective entity ${ }^{2}$ that may socially legitimate a person's sickness. ${ }^{5}$ A biomedical diagnosis may also transfer responsibility for cure from patient to health professional. ${ }^{1,6}$ However, when a patient's illness cannot be visualized and explained by pathology, doctors may attribute it to psychological or social problems. ${ }^{7}$ The patient may then be expected to take responsibility for managing the problems him- or herself. However, this may not reflect the patient's own perceptions and expectations. Patients can become dissatisfied with health services and adopt personal strategies, and sometimes collective politicized movements, to reshape meanings of diagnoses. ${ }^{1}$

Much writing on diagnosis has been from a biomedical perspective, but there has recently been renewed interest in the sociology of diagnosis. ${ }^{1,8}$ A number of sociological perspectives can be applied to diagnosis. From a labelling perspective, a diagnosis can be seen as a status applied 'externally' by others to an individual's behaviour. ${ }^{9}$ This status, of which the individual is a largely passive recipient, tends to be irreversible, and may reinforce the very behaviour that occasioned its application (by analogy with the notion of secondary deviance). In contrast to this view, the negotiated order - a perspective based in symbolic interactionism ${ }^{10}$ - takes a less deterministic view of diagnosis and sees it as the result of a process of negotiation between the practitioner and the patient, in which both parties can exert influence. ${ }^{11}$ Whereas the doctor is able to apply the diagnosis, the patient is free either to accept or to reject it. More broadly, diagnoses can be seen purely as social constructs; the conditions that they denote do not have an independent objective status, but are given diagnostic labels through a sociopolitical process. ${ }^{6,12}$ This fundamentally social character of diagnoses highlights, and makes sense of, two phenomena. First, the notion of being ill without any evident pathology causes the objective status of diagnosis to be questioned, and certain diagnoses can thereby become socially contested. ${ }^{1}$ Second, naming problems of everyday living and deviant behaviours with a diagnosis may set in motion a medicalization of people's lives. ${ }^{13}$ 
Fibromyalgia syndrome (FMS) is often used as an example of a contested disorder. It is a prevalent chronic musculoskeletal condition, characterized by widespread pain and excessive fatigue, together with an array of other complaints. In 1990, the American College of Rheumatology (ACR) classification criteria for FMS were published. ${ }^{14}$ The same year, the diagnosis was recognized by the World Health Organization and included in version 10 of the International Classification of Diseases under the chapter non-articular rheumatism with unknown etiology. ${ }^{15}$ The ACR-1990 classification criteria include reported pain of at least three months duration and present in at least three body quadrants and the axial skeleton, together with excessive tenderness by moderate pressure on at least 11 of 18 defined spots throughout the body. ${ }^{14}$ Originally, the tender points were considered to anchor pain to biological abnormalities. ${ }^{16}$ Today, the diagnosis of FMS is explained by an abnormal amplification of stimuli in the central nervous system. ${ }^{17}$ However, such changes cannot be confirmed by clinical examination. ${ }^{15}$ Thus, although FMS is often explained by biological alterations, the diagnostic criteria do not fully accord with the characteristics of a biomedical diagnosis.

Over the years, the FMS diagnosis has been the subject of numerous debates among scientists and clinicians. In particular, a controversy over the role of tender points has arisen due to their lack of specificity (also found in pain-free individuals) and validity (uncertain relationship to pathogenesis). ${ }^{15}$ Thus, in the new ACR-2010 criteria the tender point examination has been removed, and presently the diagnostic criteria comprise a cluster of symptoms. ${ }^{18}$ This separates the diagnosis further from a biomedical diagnostic category. Another debate has centred on the clinical implications of giving patients a diagnosis of FMS. For example, the diagnosis has been argued to set in motion a search for medical help that does not exist, ${ }^{19}$ and to medicalize people's psychosocial problems. ${ }^{20}$ These debates, however, have not been appreciably informed by how patients themselves perceive the diagnosis, though some qualitative studies have described patients' diagnostic experiences. ${ }^{21}$ In order to explore more fully the role of diagnosis in individuals' experience and understanding of FMS, our aim was to carry out a metasynthesis to examine in greater detail how patients experience the process and consequences of receiving a diagnosis of FMS.

\section{Methods}

\section{Design}

Metasynthesis of qualitative studies is increasingly used to gain an understanding of individuals' perspectives on their illness. A metasynthesis aims to synthesize findings across qualitative studies, and seeks new insights beyond those of individual studies. ${ }^{22}$ The term refers to a family of different methods of synthesis. ${ }^{22-24}$ In a recent text, the authors sort the various approaches according to their having either aggregative or interpretive purposes. ${ }^{22}$ The form of metasynthesis applied here is the interpretive approach of meta-ethnography, originally described by Noblit and Hare. ${ }^{24}$ A key element in meta-ethnography is translation. ${ }^{25}$ This involves an analytical transfer of concepts and insights between studies. In the process, recurrent or shared concepts - and points of similarity (reciprocal translation) or difference (refutational translation) in such concepts - are identified across studies and explicated in an 
iterative manner. In parallel, an overarching process of lines-of-argument synthesis seeks to discover 'a whole among a set of parts', ${ }^{24}$ p63 allowing an understanding to be constructed that builds upon, and is greater than, that contained within the individual studies.

\section{Literature search and study selection}

A systematic search was carried out in Medline ( $n=562)$, PsychInfo $(n=430)$, Cinahl $(n=290)$, AMED ( $n=95)$, and Social Science Citation Index ( $n=486)$ up to May 2016, supplemented by the authors' knowledge of the literature. The search terms are given in Table 1. After duplicates had been excluded, 1194 titles and abstracts were independently read by two of the authors (BA, AMM). During this reading we excluded more duplicates, quantitative studies, studies addressing chronic pain with no specification of diagnosis or including diagnoses additional to FMS, editorials, reviews, conference reports and dissertations. Decisions regarding the inclusion/exclusion of papers were then compared, and in the event of disagreement the abstracts were re-read, and if necessary the full papers were read, and discussed. After this process, 93 papers examining subjective experiences of patients with FMS were subjected to a close reading of the full text to identify whether they included information about patients' diagnostic experiences. Twenty-six papers were thereby included in the metasynthesis, and in addition one book chapter ${ }^{26}$ and one book. ${ }^{27}$

Table 1. Literature search terms

Search terms were: fibromyalgia AND qualitative OR lived OR life OR living OR interview* OR narrative* OR narration* OR semi structured OR thematic OR focus OR open ended OR grounded OR emic OR etic OR hermeneutic* OR semiotic* OR data saturation OR social OR post structural* OR poststructural* OR cooperative inquir* OR co operative inquir* OR humanistic OR existential OR experiential OR paradigm OR field OR ethnonursing OR action research OR observ* OR phenomenol* OR subjective OR story OR stories OR experience*.

\section{Quality appraisal}

Among the appraisal tools developed for metasynthesis, we considered the QUARI developed by the Joanna Briggs Institute to address appropriate domains whilst avoiding specific philosophical or methodological assumptions inapplicable to certain studies. ${ }^{28}$ We selected five of the QUARI criteria to appraise the quality of primary studies (Table 2). Items 2-4 were applied to assess methodological coherence and consistency, and items 1 and 5 were rephrased to evaluate how authors came to find meaning in informants' accounts. After piloting, we made certain modifications by operationalizing our interpretations of the specific items to align them with our interpretivist perspective (Table 2). The reports were assessed independently by two authors (JS, AMM) with respect to whether each criterion was met, partially met, or not met; any disagreements were resolved through discussion. No attempt was made to assign numerical scores, nor was the quality appraisal used to determine the inclusion of studies. 
Table 2. Operationalization of the appraisal criteria

\begin{tabular}{|l|l|}
\hline Criterion & Operationalization \\
\hline $\begin{array}{l}\text { 1. Is there a statement as to the } \\
\text { researchers' philosophical and/or } \\
\text { theoretical perspective? }\end{array}$ & $\begin{array}{l}\text { Does the report clearly articulate the philosophical or } \\
\text { theoretical premises on which the study is based? [The } \\
\text { philosophical/ theoretical position adopted should be } \\
\text { identifiable separately from the methodological } \\
\text { approach] }\end{array}$ \\
\hline $\begin{array}{l}\text { 2. Is there congruity between the } \\
\text { research methodology and the } \\
\text { research question or objectives? }\end{array}$ & $\begin{array}{l}\text { Is the study methodology appropriate for addressing } \\
\text { the research question? }\end{array}$ \\
\hline $\begin{array}{l}\text { 3. Is there congruity between the } \\
\text { research methodology and the } \\
\text { methods used to collect data? }\end{array}$ & $\begin{array}{l}\text { Are the data collection methods appropriate to the } \\
\text { methodology? }\end{array}$ \\
\hline $\begin{array}{l}\text { 4. Is there congruity between the } \\
\text { research methodology and the } \\
\text { representation and analysis of data? }\end{array}$ & $\begin{array}{l}\text { Are the data analyzed and represented in ways that are } \\
\text { congruent with the stated methodological position? }\end{array}$ \\
\hline $\begin{array}{l}\text { 5. Is there a reflexive focus on the } \\
\text { relationship between the researcher } \\
\text { and the research process? }\end{array}$ & $\begin{array}{l}\text { Are the potential for the researcher to shape the study, } \\
\text { and the potential of the research process itself to } \\
\text { shape the researcher's interpretations, acknowledged } \\
\text { and addressed? }\end{array}$ \\
\hline
\end{tabular}

\section{Synthesis and analysis}

Information from each report was entered in a grid, which included identifying information for the report, study aims, theoretical perspective (if stated), methods of analysis, main findings, and extracts of findings, and columns for coding. Initially, each publication was read several times to get a general overview of issues relating to diagnosis emerging across the reports. This reading revealed that informants' perceptions and experiences relating to the diagnosis evolved in the sequential process of searching for a diagnosis, arriving at a diagnosis, and living with the diagnosis. The extracted findings were sorted accordingly. In meta-ethnography, themes, concepts and metaphors expressing the primary authors' interpretations are the data for analysis. However, interpretations of diagnostic experience were often neither expressed in themes nor explicitly interpreted in the discussion, as the studies' research questions focused on illness experiences in general. Thus, both descriptive and interpretive constructs in the results sections were extracted, and broader concepts encapsulating their meaning were identified and applied in further analysis. When no specific concept was given, we examined whether those used by other authors could explain the findings of the particular study. Common or recurring concepts identified in the reports - such as disbelief, skepticism, feeling degraded, humiliated, and symptoms explained as imagined, trivial, and psychological - were compared to determine ways in which they might translate into each other. ${ }^{24}$ Refutational translation was also attempted. However, the concepts were broadly similar across reports, and when they appeared to differ they could be translated into broader, overarching concepts, such as validation, meaning-making, and legitimation. Based on this systematic process of reading and analysis, the findings were taken together in a lines- 
of-argument synthesis that led us to understand that, from the patient's point of view, the diagnosis had two main overarching, intertwined purposes: 1 . To validate and make sense of the individual's illness experience, and 2. To legitimate the illness in the broader social world.

\section{Findings}

\section{Studies and methodological appraisal}

Table 3 gives an overview of the publications included. The reports refer to interviews with 475 informants diagnosed with FMS (450 women and 25 men; 247 from Europe, 200 from North America, 13 from Asia, 15 from Africa), aged between 16 and 80 years. The time since diagnosis of FMS ranged from 1 week up to 20 years. Informants were recruited from clinics, patient associations, patient support groups, or through advertising and snowball sampling. Five studies focused on examining patients' diagnostic experience, ${ }^{27,29-32}$ and in the others, patients' diagnostic experiences were embedded in accounts of their experiences of living with FMS. Face-to-face interview studies predominated, though email interviews and focus groups were used in some studies. Most studies met at least three quality appraisal criteria, and five met all criteria. The criteria most commonly unfulfilled were those relating to the researchers' philosophical and theoretical stance and issues concerning reflexivity (criteria 1 and 5, respectively; Table 2).

\section{Substantive findings}

Analysis of the studies resulted in two broad concepts relating to the role of the diagnosis in validating and making sense of bodily experiences, and its role in legitimating sickness. These concepts emerged in relation to other concepts that characterized the diagnostic trajectory (Fig. 1). The presentation of findings is organized in relation to the first set of concepts. 
Table 3. Overview of the studies included in the metasynthesis

\begin{tabular}{|c|c|c|c|c|c|c|c|c|c|}
\hline \multirow[b]{2}{*}{ Study } & \multirow[b]{2}{*}{ Country } & \multirow{2}{*}{$\begin{array}{l}\text { Stated } \\
\text { theoretical or } \\
\text { philosophical } \\
\text { perspective }\end{array}$} & \multirow[b]{2}{*}{$\begin{array}{l}\text { Recruitment } \\
\text { source }\end{array}$} & \multicolumn{2}{|r|}{ Sample } & \multicolumn{2}{|c|}{ Description of methodology } & \multicolumn{2}{|c|}{$\begin{array}{c}\text { Methodological } \\
\text { appraisal criteria* }\end{array}$} \\
\hline & & & & Sex & Characteristics & Data collection & Data analysis & $\begin{array}{c}\text { Not } \\
\text { fulfilled }\end{array}$ & $\begin{array}{l}\text { Partially } \\
\text { fulfilled }\end{array}$ \\
\hline $\begin{array}{l}\text { Armentor } \\
2016^{54}\end{array}$ & USA & $\begin{array}{l}\text { Interactionism, } \\
\text { ethnomethod- } \\
\text { ology \& } \\
\text { constructionist } \\
\text { perspectives }\end{array}$ & $\begin{array}{l}\text { Flyer in } \\
\text { rheumatology \& } \\
\text { counselling } \\
\text { offices; snowball } \\
\text { sampling }\end{array}$ & $20 \mathrm{~F}$ & $\begin{array}{l}\text { Age: } 32-80 y \\
\text { Time since diagnosis: } \\
\text { mean of } 12 y\end{array}$ & $\begin{array}{l}\text { Thematic individual } \\
\text { in-depth interviews }\end{array}$ & $\begin{array}{l}\text { Grounded theory } \\
\text { Open line-by-line } \\
\text { coding \& } \\
\text { comparative analysis }\end{array}$ & - & - \\
\hline $\begin{array}{l}\text { Arnold et al } \\
2008^{44}\end{array}$ & $\begin{array}{l}\text { Canada } \\
\text { USA }\end{array}$ & Not described & $\begin{array}{l}\text { Community- \& } \\
\text { university-based } \\
\text { rheumatology } \\
\text { practices }\end{array}$ & $48 \mathrm{~F}$ & $\begin{array}{l}\text { Age: } 31-72 y \\
\text { Time since diagnosis: } \\
1-18 y\end{array}$ & $\begin{array}{l}\text { Focus group } \\
\text { interviews }\end{array}$ & $\begin{array}{l}\text { Grounded theory } \\
\text { according to Corbin } \\
\text { \& Strauss }\end{array}$ & 1,5 & - \\
\hline Barker $2005^{27}$ & USA & $\begin{array}{l}\text { Sociological } \\
\text { theories }\end{array}$ & $\begin{array}{l}\text { Patient } \\
\text { conference \& } \\
\text { FMS support } \\
\text { groups }\end{array}$ & $\begin{array}{l}30 F \\
4 M\end{array}$ & Age: $26-65 y$ & $\begin{array}{l}\text { In-depth individual } \\
\text { interviews; focus } \\
\text { group interviews }\end{array}$ & Not given & 4 & - \\
\hline $\begin{array}{l}\text { Briones- } \\
\text { Vozmediano et } \\
\text { al } \\
2013^{\# 29}\end{array}$ & Spain & Not described & $\begin{array}{l}\text { Patient } \\
\text { associations }\end{array}$ & $9 \mathrm{~F}, 3 \mathrm{M}$ & Age: 29-61y & $\begin{array}{l}\text { Thematic individual } \\
\text { interviews }\end{array}$ & $\begin{array}{l}\text { Descriptive \& } \\
\text { interpretive } \\
\text { discourse analysis }\end{array}$ & 1 & - \\
\hline $\begin{array}{l}\text { Colmenares-Roa } \\
\text { et al } 2016^{33}\end{array}$ & Mexico & $\begin{array}{l}\text { Critical } \\
\text { anthropological } \\
\text { perspective }\end{array}$ & $\begin{array}{l}\text { Rheumatologists' } \\
\text { FMS clinic in a } \\
\text { public hospital \& } \\
\text { private clinic }\end{array}$ & $5 F, 3 M$ & $\begin{array}{l}\text { Age: } 34-74 y \\
\text { Symptoms: 1-10y }\end{array}$ & $\begin{array}{l}\text { Hospital } \\
\text { ethnography - } \\
\text { fieldwork \& in-depth } \\
\text { interviews }\end{array}$ & Narrative analysis & 5 & - \\
\hline $\begin{array}{l}\text { Cooper \& } \\
\text { Gilbert } 2016^{34}\end{array}$ & $\begin{array}{l}\text { South } \\
\text { Africa }\end{array}$ & Not described & $\begin{array}{l}\text { Snowball } \\
\text { sampling through } \\
\text { peer networks }\end{array}$ & $15 \mathrm{~F}$ & Age: $23-59 y$ & $\begin{array}{l}\text { In-depth semi- } \\
\text { structured interviews }\end{array}$ & Thematic analysis & 1,5 & - \\
\hline $\begin{array}{l}\text { Cunningham \& } \\
\text { Jillings } 2006^{53}\end{array}$ & Canada & Not described & $\begin{array}{l}\text { Self-referrals } \\
\text { through faculty } \\
\text { newsletter }\end{array}$ & $7 \mathrm{~F}, 1 \mathrm{M}$ & $\begin{array}{l}\text { Age: } 30 y-l a t e 70 s \\
\text { Time since diagnosis: } \\
1.5-13 y\end{array}$ & $\begin{array}{l}\text { In-depth, semi- } \\
\text { structured individual } \\
\text { interviews }\end{array}$ & $\begin{array}{l}\text { Constant } \\
\text { comparative analysis }\end{array}$ & 1 & - \\
\hline
\end{tabular}




\begin{tabular}{|c|c|c|c|c|c|c|c|c|c|}
\hline $\begin{array}{l}\text { Dennis et al } \\
2013^{47}\end{array}$ & UK & Not described & $\begin{array}{l}\text { Online \& real- } \\
\text { world support } \\
\text { groups }\end{array}$ & $\begin{array}{l}17 \mathrm{~F} \\
3 \mathrm{M}\end{array}$ & $\begin{array}{l}\text { Age: } 18-64 y \\
\text { Symptoms: } \geq 2 y \\
\text { Time since diagnosis: } \\
6 m-10 y\end{array}$ & $\begin{array}{l}\text { E-mail dialogic } \\
\text { interview responding } \\
\text { to } 40 \text { open-ended } \\
\text { questions }\end{array}$ & $\begin{array}{l}\text { Interpretative } \\
\text { phenomenological } \\
\text { analysis }\end{array}$ & 1,5 & - \\
\hline Diver $2013^{26}$ & UK & $\begin{array}{l}\text { Frank's } \\
\text { narrative } \\
\text { typologies }\end{array}$ & $\begin{array}{l}\text { Rheumatology } \\
\text { clinic }\end{array}$ & $\begin{array}{l}22 \mathrm{~F} \\
1 \mathrm{M}\end{array}$ & $\begin{array}{l}\text { Age: } 25-71 \\
\text { Time to diagnosis: } 0.5- \\
5 y\end{array}$ & $\begin{array}{l}\text { In-depth semi- } \\
\text { structured interviews }\end{array}$ & $\begin{array}{l}\text { Narrative thematic } \\
\text { analysis }\end{array}$ & 5 & - \\
\hline $\begin{array}{l}\text { Durif-Bruckert } \\
\text { et al } 2014^{43}\end{array}$ & France & $\begin{array}{l}\text { Anthropological } \\
\text { \& psychological } \\
\text { theories; } \\
\text { knowledge } \\
\text { negotiation } \\
\end{array}$ & $\begin{array}{l}\text { Internal medicine } \\
\& \text { rheumatology } \\
\text { hospital units }\end{array}$ & $\begin{array}{l}32 \mathrm{~F} \\
3 \mathrm{M}\end{array}$ & $\begin{array}{l}\text { Age: } 25-70 y \\
\text { Symptoms: } 6 \mathrm{~m}-30 y \\
\text { Time since diagnosis: } \\
\leq 1 \mathrm{~d}-15 \mathrm{y}\end{array}$ & $\begin{array}{l}\text { Semi-structured } \\
\text { individual interviews }\end{array}$ & Content analysis & 5 & - \\
\hline $\begin{array}{l}\text { Hallberg \& } \\
\text { Carlsson } 1998^{45}\end{array}$ & Sweden & Interactionism & $\begin{array}{l}\text { Specialized } \\
\text { hospital units to } \\
\text { consider disability } \\
\text { pension }\end{array}$ & $22 \mathrm{~F}$ & Age: 22-60y & $\begin{array}{l}\text { Individual open- } \\
\text { ended interviews }\end{array}$ & $\begin{array}{l}\text { Constant comparison } \\
\text { analysis according to } \\
\text { Grounded theory }\end{array}$ & 1,5 & - \\
\hline $\begin{array}{l}\text { Hellström et al } \\
1999^{35}\end{array}$ & Sweden & $\begin{array}{l}\text { Phenomenology } \\
\text { \& psychological } \\
\text { theories }\end{array}$ & $\begin{array}{l}\text { Patient } \\
\text { Association } \\
\text { meetings }\end{array}$ & $9 \mathrm{~F}, 1 \mathrm{M}$ & $\begin{array}{l}\text { Age: } 32-50 y \\
\text { Symptoms: 4-18y } \\
\text { Time since diagnosis: } \\
2-7 y\end{array}$ & Individual interviews & $\begin{array}{l}\text { Karlsson's } \\
\text { interpretative } \\
\text { phenomenological } \\
\text { analysis }\end{array}$ & 1,5 & - \\
\hline $\begin{array}{l}\text { Henriksson } \\
1995^{\# 39}\end{array}$ & $\begin{array}{l}\text { Sweden } \\
\& \text { USA }\end{array}$ & Not described & Outpatient clinics & $40 \mathrm{~F}$ & $\begin{array}{l}\text { Age: } 16-57 y \\
\text { Duration: } 0.5-40 y\end{array}$ & $\begin{array}{l}\text { Semi-structured } \\
\text { interviews by } \\
\text { Occupational Case } \\
\text { Analysis Interview }\end{array}$ & $\begin{array}{l}\text { Qualitative content } \\
\text { analysis }\end{array}$ & 1,5 & \\
\hline $\begin{array}{l}\text { Homma et al } \\
2016^{48}\end{array}$ & Japan & $\begin{array}{l}\text { Hermeneutic } \\
\text { phenomenology }\end{array}$ & $\begin{array}{l}\text { Self-help group } \\
\text { members }\end{array}$ & $\begin{array}{l}11 \mathrm{~F} \\
2 \mathrm{M}\end{array}$ & $\begin{array}{l}\text { Age: } 29-73 y \\
\text { Symptoms: } 0.3-63 y\end{array}$ & $\begin{array}{l}\text { In-depth thematic } \\
\text { interviews }\end{array}$ & $\begin{array}{l}\text { Hermeneutic- } \\
\text { phenomenological } \\
\text { analysis }\end{array}$ & - & - \\
\hline $\begin{array}{l}\text { Lempp et al } \\
2009^{49}\end{array}$ & UK & Not described & $\begin{array}{l}\text { Rheumatology } \\
\text { outpatient clinic }\end{array}$ & $\begin{array}{l}11 \mathrm{~F} \\
1 \mathrm{M}\end{array}$ & $\begin{array}{l}\text { Age: } 20-69 y \\
\text { Illness duration: } 5 m- \\
11 y\end{array}$ & $\begin{array}{l}\text { Semi-structured } \\
\text { individual interviews }\end{array}$ & $\begin{array}{l}\text { Content analysis, } \\
\text { constant comparison } \\
\text { analysis } \\
\text { Discourse analysis }\end{array}$ & 1,5 & - \\
\hline $\begin{array}{l}\text { Madden \& Sim } \\
2006,{ }^{30} 2016^{32}\end{array}$ & UK & $\begin{array}{l}\text { Interactionism, } \\
\text { theories of } \\
\text { negotiated } \\
\text { order \& the self }\end{array}$ & $\begin{array}{l}\text { Rheumatology } \\
\text { clinic }\end{array}$ & $\begin{array}{l}16 \mathrm{~F} \\
1 \mathrm{M}\end{array}$ & $\begin{array}{l}\text { Age: } 25-55 y \\
\text { Time since diagnosis: } \\
1 w-8 \text { y }\end{array}$ & $\begin{array}{l}\text { Semi-structured } \\
\text { interviews; } \\
\text { documents }\end{array}$ & $\begin{array}{l}\text { Induction-abduction } \\
\text { method \& theory- } \\
\text { driven analysis }\end{array}$ & - & 5 \\
\hline
\end{tabular}




\begin{tabular}{|c|c|c|c|c|c|c|c|c|c|}
\hline $\begin{array}{l}\text { McMahon et al } \\
2012^{38}\end{array}$ & UK & $\begin{array}{l}\text { Narrative } \\
\text { perspective }\end{array}$ & $\begin{array}{l}\text { Multi-disciplinary } \\
\text { pain management } \\
\text { clinic }\end{array}$ & $10 \mathrm{~F}$ & $\begin{array}{l}\text { Age: } 25-70 y \\
\text { Symptoms: since } \\
\text { childhood-15y } \\
\text { Time since diagnosis: } \\
5 \mathrm{~m}-14 \mathrm{y}\end{array}$ & Narrative interviews & Narrative analysis & - & - \\
\hline $\begin{array}{l}\text { Mengshoel \& } \\
\text { Heggen } 2004^{50}\end{array}$ & Norway & $\begin{array}{l}\text { Theories of } \\
\text { illness, health \& } \\
\text { sick role }\end{array}$ & $\begin{array}{l}\text { Prior participants } \\
\text { in out-patient } \\
\text { exercise \& patient } \\
\text { education } \\
\text { programs }\end{array}$ & $5 F$ & $\begin{array}{l}\text { Age: } 37-49 y \\
\text { Time since diagnosis: } \\
1-15 y \text {, but now } \\
\text { healthy again }\end{array}$ & $\begin{array}{l}\text { Individual, open } \\
\text { interviews }\end{array}$ & Thematic analysis & - & - \\
\hline $\begin{array}{l}\text { Raymond \& } \\
\text { Brown } 2000^{40}\end{array}$ & Canada & Not described & $\begin{array}{l}\text { Patient } \\
\text { association }\end{array}$ & $6 \mathrm{~F}, 1 \mathrm{M}$ & $\begin{array}{l}\text { Age: } 38-47 y \\
\text { Symptoms: average } 8 y \\
\text { before diagnosis } \\
\text { Time since diagnosis: } \\
1-13 y\end{array}$ & $\begin{array}{l}\text { Semi-structured, } \\
\text { individual interviews }\end{array}$ & $\begin{array}{l}\text { Interpretative } \\
\text { analysis of patterns, } \\
\text { categories \& themes }\end{array}$ & 1 & - \\
\hline $\begin{array}{l}\text { Sallinen et al } \\
2012^{37}\end{array}$ & Finland & $\begin{array}{l}\text { Narrative } \\
\text { theory \& } \\
\text { constructionism }\end{array}$ & $\begin{array}{l}\text { Prior participants } \\
\text { in rehabilitation } \\
\text { program }\end{array}$ & $20 \mathrm{~F}$ & $\begin{array}{l}\text { Age: } 34-65 y \\
\text { Symptoms: } 10-30 y\end{array}$ & $\begin{array}{l}\text { Individual narrative } \\
\text { interviews }\end{array}$ & Narrative analysis & - & - \\
\hline Schaefer $2005^{46}$ & USA & $\begin{array}{l}\text { Phenomenology } \\
\text { inspired by Van } \\
\text { Manen }\end{array}$ & $\begin{array}{l}\text { Advertising in } \\
\text { newspapers }\end{array}$ & $10 \mathrm{~F}$ & $\begin{array}{l}\text { Age: } 37-59 y \\
\text { Duration of FMS: 2- } \\
18 y\end{array}$ & Individual interviews & $\begin{array}{l}\text { Van Manen's } \\
\text { phenomenological } \\
\text { method \& thematic } \\
\text { analysis }\end{array}$ & 1 & 5 \\
\hline Schaefer $1995^{36}$ & USA & $\begin{array}{l}\text { Grounded } \\
\text { theory \& } \\
\text { feminist } \\
\text { methods }\end{array}$ & $\begin{array}{l}\text { Participants in } \\
\text { community } \\
\text { programs }\end{array}$ & $36 \mathrm{~F}$ & Not described & $\begin{array}{l}\text { In-depth individual } \\
\text { interviews }\end{array}$ & $\begin{array}{l}\text { Constant } \\
\text { comparative method; } \\
\text { open, axial \& } \\
\text { selective coding }\end{array}$ & 1,5 & - \\
\hline $\begin{array}{l}\text { Sturge-Jacobs } \\
2002^{41}\end{array}$ & Canada & $\begin{array}{l}\text { Phenomenology } \\
\text { inspired by van } \\
\text { Manen }\end{array}$ & $\begin{array}{l}\text { Out-patient } \\
\text { education } \\
\text { program at } \\
\text { tertiary care }\end{array}$ & $9 \mathrm{~F}$ & $\begin{array}{l}\text { Age: } 30-56 y \\
\text { Time since diagnosis: } \\
\geq 1 y\end{array}$ & $\begin{array}{l}\text { Individual } \\
\text { unstructured } \\
\text { interviews }\end{array}$ & $\begin{array}{l}\text { Van Manen's } \\
\text { phenomenological } \\
\text { method \& thematic } \\
\text { analysis }\end{array}$ & 1,5 & - \\
\hline $\begin{array}{l}\text { Söderberg et al } \\
1999^{\# 1}\end{array}$ & Sweden & $\begin{array}{l}\text { Phenomenologi } \\
\text { cal-hermeneutic } \\
\text { method inspired } \\
\text { by Ricoeur }\end{array}$ & $\begin{array}{l}\text { Rheumatology } \\
\text { clinic }\end{array}$ & $14 \mathrm{~F}$ & $\begin{array}{l}\text { Age: } 35-50 y \\
\text { Symptoms: } 1-25 y \\
\text { Time since diagnosis: } \\
0.5-6 y\end{array}$ & $\begin{array}{l}\text { Individual interviews } \\
\text { with narrative } \\
\text { approach }\end{array}$ & $\begin{array}{l}\text { Phenomenological } \\
\text { hermeneutic analysis }\end{array}$ & 1,5 & - \\
\hline
\end{tabular}




\begin{tabular}{|c|c|c|c|c|c|c|c|c|c|}
\hline $\begin{array}{l}\text { Undeland \& } \\
\text { Malterud } 2007^{31}\end{array}$ & Norway & Not described & $\begin{array}{l}\text { Participants in } \\
\text { self-help groups }\end{array}$ & $11 \mathrm{~F}$ & $\begin{array}{l}\text { Age: } 42-67 y \\
\text { Illness duration: } 8-40 \\
\text { yrs (2-40y before } \\
\text { diagnosis) } \\
\text { Time since diagnosis: } \\
\text { 5-20y }\end{array}$ & $\begin{array}{l}\text { Focus group } \\
\text { interviews }\end{array}$ & $\begin{array}{l}\text { Systematic text } \\
\text { condensation }\end{array}$ & 1 & 5 \\
\hline $\begin{array}{l}\text { Van Gordon et } \\
\text { al } \\
2016^{52}\end{array}$ & UK & $\begin{array}{l}\text { Symbolic } \\
\text { interactionism }\end{array}$ & $\begin{array}{l}\text { Mindfulness } \\
\text { intervention }\end{array}$ & $9 \mathrm{~F}, 1 \mathrm{M}$ & $\begin{array}{l}\text { Age: } 29-64 y \\
\text { Time since diagnosis: } \\
2-11 y\end{array}$ & $\begin{array}{l}\text { Individual semi- } \\
\text { structured interviews }\end{array}$ & $\begin{array}{l}\text { Interpretative } \\
\text { phenomenological } \\
\text { analysis }\end{array}$ & 1,5 & - \\
\hline $\begin{array}{l}\text { Wuytack \& } \\
\text { Miller } 2011^{42}\end{array}$ & Belgium & $\begin{array}{l}\text { Phenomenology } \\
\text { \& Husserlian } \\
\text { transcendental } \\
\text { subjectivity }\end{array}$ & Self-help group & $6 F$ & $\begin{array}{l}\text { Age: } 36-66 y \\
\text { Symptoms before } \\
\text { diagnosis: } 1-19 y \text { Time } \\
\text { since diagnosis: } 1-9 y\end{array}$ & $\begin{array}{l}\text { Semi-structured } \\
\text { individual interviews }\end{array}$ & $\begin{array}{l}\text { Coding of themes \& } \\
\text { categories, \& } \\
\text { identifying patterns } \\
\text { \& dimensions }\end{array}$ & 1,5 & - \\
\hline
\end{tabular}

Figure 1. Diagnostic trajectory: themes and subthemes.

\begin{tabular}{|l|}
$\begin{array}{l}\text { Long and strenuous } \\
\text { search for a } \\
\text { diagnosis }\end{array}$ \\
Role of the diagnosis in validating and making sense of bodily experiences \\
- Endeavouring to confirm the reality of the illness \\
- Diagnosis has ambiguous validity and meaning \\
- Diagnosis of uncertain validator of sickness to others \\
- Diagnosis affects credibility and dignity \\
- Questioning the diagnosis and medical authority \\
validity of the \\
diagnosis
\end{tabular}

Themes and subthemes 


\section{Role of FMS diagnosis in validating and making sense of bodily experiences}

\section{Endeavouring to confirm the reality of the illness}

In the pre-diagnostic period, informants' main concern was to make sense of their pain and fatigue with help of the physician's technical competency. ${ }^{33,34}$ The onset of pain was described sometimes as sudden without any apparent warning, ${ }^{35,36}$ dating from a clearly identifiable time, ${ }^{32}$ or sometimes as insidious. ${ }^{37}$ Informants attributed the acute onset to significant, unpredictable life events, ${ }^{35}$ physical or psychological trauma, or as something dormant being triggered within the body. ${ }^{30}$ The gradually developing symptoms were often ignored until they became so debilitating that they could no longer be considered temporary ${ }^{37}$ or as related to work overload, age, flu, or comorbid illnesses. ${ }^{26,35}$ When the illness experience became unmanageable and could not be explained, and individuals' lay networks confirmed that something had to be wrong, they consulted a physician and medical investigations were performed. ${ }^{32}$ However, ordinary laboratory and imaging assessments typically did not reveal any pathology, and informants were referred for further examinations to explore whether they suffered from other diseases such as progressive neurological or rheumatic disorders. ${ }^{29,36,38,39}$ Often, this meant a merry-go-round to various specialists and other practitioners without receiving a diagnosis, ${ }^{31,32,34,36,38,40-42}$ or receiving a different diagnosis from FMS. ${ }^{43}$ Whilst these new consultations raised expectations of an answer as to what was wrong, this initial hope was frequently replaced by disappointment. . $^{2939-41}$

Frequently, several years were spent in such attempts to exclude other diseases. $^{29,34,37,38,42-44}$ Meanwhile, informants were often told by their physician that their illness had to be imagined or psychological. ${ }^{30,35,36,38-43,45,46}$ However, although psychological and social factors might accord with informants' own initial explanations, they expected something more to be revealed by medical investigations. ${ }^{32}$ On finally being diagnosed with FMS, informants described a great sense of relief. . $^{27,31,38-42,45-52}$ The diagnosis signified that they were not suffering from an organic disease, did not risk ending up in a wheelchair, and were not mentally ill. ${ }^{29,31,39,45,47-51}$ Furthermore, informants had a name to which they could relate their suffering, and which they could communicate to others. ${ }^{27,31,38}$ Having the label of FMS, ${ }^{40,42,51}$ receiving prescriptions, sick notes or referrals to other health professionals, ${ }^{45}$ and an awareness of a shared destiny with other patients, ${ }^{30,31}$ all contributed to validating their experiences as real.

\section{FMS diagnosis has ambiguous validity and meaning}

The immense immediate relief of getting a diagnosis faded over time as informants realized with disappointment that it was not accompanied by any curative treatment and their life was likely to be permanently altered. . $7,30,31,38,47,48,50$ Their next pressing question was what could be done to deal with the illness; the search for a diagnosis was now replaced by a trial-anderror process to find effective treatment and more appropriate coping strategies. $33,40,43$ Individuals also had to find meaning in the diagnosis. ${ }^{30,33,51}$ Thus, initial uncertainty as to the nature of the diagnosis was now replaced by uncertainty as to what the FMS diagnosis meant and how to manage it. ${ }^{32,33,40}$ For some, however, the diagnosis had more positive consequences - for example, likened to having won a lottery ${ }^{45}$ - and others feared losing the 
diagnosis they had spent so long to achieve if they became much better. ${ }^{52}$ For others, a problematic diagnosis was preferable to no diagnosis at all. ${ }^{27}$

After trying various therapies without success, some informants found that their physicians lost interest in them or, as in the time prior to diagnosis, trivialized their pain as a normal part of life, or suggested that FMS was psychological in nature. ${ }^{35,48,50,53,54}$ A number of informants had become aware, directly or indirectly, of the ambivalent attitudes of health professionals towards FMS, in particular their skepticism as to its being a 'real' condition, and their hesitancy in using the diagnosis. ${ }^{31,33,34,54}$ This ambivalence on the part of health professionals often caused confusion among informants and led them to doubt their own experiences; they began to ask themselves whether their illness was in fact imagined or simply an overreaction to something essentially normal. ${ }^{30,36,41,42,48,54}$ In contrast, some informants actively resisted being viewed as a malingerer during consultations ${ }^{41}$ and, in a process of negotiation with their physicians, advanced their own interpretations of their experience. ${ }^{32}$ Predominantly, the FMS diagnosis suggested a permanently altered life, ${ }^{38,40,42}$ but nevertheless not all were pessimistic about their future, ${ }^{42}$ and some were able to redefine FMS from a chronic to a temporary condition in response to physicians' comments that they had seen patients who had become healthy again. ${ }^{50}$

\section{Role of FMS diagnosis in legitimating sickness}

\section{FMS diagnosis as an uncertain validator of sickness to others}

The diagnosis had an important function to legitimate individuals' illness to those around them, ${ }^{34,41}$ serving to counter skepticism and negative attitudes on the part of others ${ }^{51}$ and to reincorporate the individual within the social world. ${ }^{27}$ The FMS label justified informants spending time on self-care ${ }^{44}$ and, at least partially, released them from social demands and responsibilities. ${ }^{45}$ Although managers could be convinced that individuals needed time for adjustment to their working situation and medical appointments, the diagnosis was nonetheless hidden in some cases for fear of losing employment. ${ }^{47}$ Informants also experienced negative comments from work colleagues, who indicated that they were not trying hard enough to combat their illness, or implicitly accused them of taking a free ride. ${ }^{38,39,46}$ Likewise, whilst family and friends could be of great support and readily share domestic responsibilities, ${ }^{34,37,54}$ some nonetheless expressed doubt regarding the illness. ${ }^{31,36}$

The FMS diagnosis did not convey a clear meaning to others and did not therefore assist informants in explaining what was wrong; ${ }^{30,31,35-38,41,42,46,48-50,54}$ they therefore felt obliged to provide further explanations. ${ }^{31}$ Like health professionals, other people questioned the reality of the illness, and informants had heard statements that someone looking so well could not be sick. . $7,31,36,38,39,41,44,51$ Thus, their bodily experience was invisible on two counts: owing to a lack of detectable internal pathology it was invisible to the medical gaze, and by virtue of the lack of obvious external manifestations of illness it was invisible to others. ${ }^{54}$ Informants therefore received varying levels of acceptance and support. ${ }^{26,42,44,50}$ However, speaking about FMS directly and in specific terms - and thereby providing an authoritative account of the illness - could lead to greater understanding and sensitivity on the part of others. $^{54}$ 
FMS diagnosis affects personal credibility and dignity

In the clinical encounter, individuals struggled to convince physicians that their illness was not imaginary or psychological, in order to become a worthy patient. ${ }^{32,35,41,51}$ When their physicians related their symptoms to psychological problems, informants felt blamed for their own suffering. ${ }^{36}$ Similarly, when accused - indirectly or directly - of being lazy or work-shy, informants perceived that their moral character was being impugned, ${ }^{46,50}$ and they were anxious that unsuccessful efforts to get better would cause health professionals and others to question whether they had the character and motivation needed to recover. ${ }^{49,50}$ Some felt that they were regarded as a 'difficult patient', 45 and others sought out 'fibro-friendly' physicians. ${ }^{27,34}$ On account of such questioning of their diagnosis and attempts to relate their suffering to psychological problems, some informants felt humiliated by health professionals $^{34,37,43,51}$ and within their social network. . $^{39,48,53}$

Informants were also aware of negative attributions of $\mathrm{FMS}^{31,51}$ - for example, to hypochondria, ${ }^{47}$ a 'women's disorder', hysteria, or simply as something everyone has. ${ }^{31}$ In the face of these negative connotations of the diagnosis, informants frequently found it hard to counteract others' distrust and stigmatizing attitudes. ${ }^{39,45,46,48,50,53}$ As a result, some doubted their own credibility and began to question the reality of their own experiences. ${ }^{50}$ Others rejected the medical diagnosis, with its negative associations, and instead constructed their own meaning of the diagnosis based on their own experience. ${ }^{30,50}$ Strategies to maintain their credibility as persons could be to attribute FMS to their perfectionism and overactive life style when explaining their illness to others, ${ }^{35,48}$ or to keep the diagnosis hidden, so as to avert stigmatization. ${ }^{47}$ To avoid being a burden to their family, they might hide their suffering and preserve a healthy image. ${ }^{46,50}$ Accordingly, individuals with FMS had to manage living with an inexplicable, invisible illness, without effective therapy, in the face of other's negative attitudes or behaviour, whilst maintaining dignity and a positive self-image.

\section{Questioning the FMS diagnosis and medical authority}

Initially, the FMS diagnosis was acceptable to informants, as it confirmed and validated their pain experience. ${ }^{30,33}$ Some informants found the tender point examination convincing and admired the rheumatologists who had thereby determined the diagnosis when so many other specialists had failed to do so. Subsequently, however, they often found the diagnostic process less satisfactory. FMS appeared to be an 'empty' diagnosis that did not materially assist their understanding of their illness, and lacked clear implications for treatment or means of coping with symptoms. ${ }^{30,33}$ Moreover, informants' skepticism about diagnosis increased when new symptoms arose and their physician tended to attach all such symptoms to the FMS diagnosis, even without examining them. ${ }^{35,37,38}$ Consequently, informants felt that FMS was a 'wastebasket' diagnosis that could accommodate any symptom. ${ }^{30,37}$ For some, this was illogical and they suspected that the diagnostic label was applied by physicians to keep them quiet. ${ }^{38}$ Informants started to question their doctors' competence and expertise, ${ }^{34,38,43,51}$ and their initial faith in the biomedical view of illness embodied in the medical diagnosis began to be eroded. ${ }^{32}$ Acceptance of the superiority of medical knowledge was replaced by an assertion of the individual's own expertise, based on a unique understanding of his or her own body, 
which could lead to a rejection of the medical diagnosis, ${ }^{32}$ and a consequent loss of faith in medical authority more broadly. ${ }^{30}$

\section{Summative interpretation and reflection}

The present analysis shows that, throughout the phases of the diagnostic trajectory, the diagnosis had two intertwined purposes: to validate and make sense of individuals' illness experience, and to legitimate their being sick in social contexts. After a long time undergoing medical examinations to exclude various diseases, it was a great relief finally to arrive at a diagnosis and feel legitimated as sick. However, when time passed and therapies proved ineffective, informants were met with skepticism by health professionals and other people, and this legitimacy waned. The diagnostic experience in FMS thereby reflects in several respects that of other contested illnesses.,7,55-59 For example, the long, frustrating prediagnostic period of consulting with various medical specialists without finding anything wrong characterizes chronic fatigue syndrome ${ }^{56}$ and repetitive strain injury. ${ }^{58}$ Swoboda's ${ }^{60}$ survey of diagnostic practice in medically contested disorders found that physicians tend first to eliminate other diseases, then test particular treatments, and thereafter perform a psychological evaluation. Such an approach to diagnosis was also displayed in many of the studies in the present synthesis.

On finally obtaining the diagnosis, informants were greatly relieved not to be suffering from a fatal disease. Thus, this sense of relief related to what they were not suffering from, rather than to an understanding of what the FMS diagnosis actually meant. At this time, informants found that the diagnosis, and physicians' prescribing therapies and providing sick notes, validated their illness and consequently legitimated them socially as a sick person. In line with a common expectation that a diagnosis is accompanied by therapy, ${ }^{2}$ they hoped finally to receive appropriate treatment. However, such therapies were ineffective and the studies revealed that, reflecting Swoboda's ${ }^{60}$ findings, physicians started to perform psychological evaluations. Discovering problematic social and personal circumstances can be a way for physicians to ascribe medical legitimacy to a patient's illness. ${ }^{57}$ However, the present informants perceived it differently; they interpreted the psychological evaluation or the trivializing of their symptoms as indications that the physician doubted that they were ill and speculated that they were abusing the FMS diagnosis. As in Aronowitz's ${ }^{6157-83}$ study of another contested diagnosis, Lyme syndrome, the legitimacy of illness was more at threat from others' questioning and distrust, both within and outside medical encounters, than from a lack of objective evidence of disease.

Sick role theory claims that those who are ill should seek qualified help and cooperate with health professionals to speed their recovery. ${ }^{62}$ The sick role is therefore conditionally legitimate, insofar as a sick person follows medical advice and adheres to appropriate prescribed treatment. ${ }^{63}$ So when informants told their physicians that they had not responded to prescribed therapies and were still unable to fulfil daily obligations, they found that their efforts to get better were questioned. Frank ${ }^{64}$ has argued that becoming ill is a moral event, and alongside the social responsibility ascribed by the sick role, patients have a moral responsibility to do what is medically 'correct' ${ }^{65}$ Consequently, informants were met with 
skepticism by others regarding the reality of their illness, or accusations of malingering or lacking moral character. In the face of such negative reactions, they struggled to maintain a credible moral self. Zavestoski ${ }^{66}$ suggests that health professionals should better understand how diagnosis and subsequent clinical action have social consequences in terms of shaping patients' social identities.

In the absence of objective medical evidence of illness, and thereby the moral imprimatur provided by a diagnosis, patients' moral credibility depends on their ability to present a convincing story to validate and legitimize illness experiences to others, in the face of either trivialization or delegitimation. ${ }^{56}$ In this situation, patients rely on their own ability to narrate their illness experiences. However, stories told by patients with chronic unexplained symptoms can be ambiguous and chaotic ${ }^{67}$ - what Bülow ${ }^{68}$ calls a 'broken narrative' - and are therefore difficult for others to fully understand. Accordingly, individuals may not succeed in preserving their moral self in the face of illness. Without a medical explanation, they have to find alternative ways to reestablish coherence between the experiences of the past and the present, as well as future expectations. ${ }^{69}$ Health professionals can play a role in co-authoring a coherent and meaningful narrative. ${ }^{2,70}$ Instead, informants felt misjudged and 'not taken seriously', and started to doubt the physicians' competency. Thus, the communication associated with the diagnostic process in FMS may put the credibility and authority both of patients and of medical expertise at stake.

The limited role of diagnosis in making sense of illness and providing social legitimacy for patients with FMS is also shown in a prior metasynthesis of the experience of FMS, ${ }^{21}$ and in another examining various chronic musculoskeletal pain syndromes, including FMS. ${ }^{71}$ These syntheses addressed patients' illness experiences broadly, whereas we examined a particular aspect of the illness experience. We thereby obtained more specific and detailed insights. Additionally, we could explore how informants' experiences varied over time, and diagnostic experiences thereby emerge as a complex and subtle process, characterized by a number of tensions, such as the following. Individuals strive to obtain the diagnosis, but then find it unsatisfactory in terms of the meaning that it provides for their illness. The diagnosis, once achieved, provides some legitimation of the illness, but the invisibility of FMS and individuals' difficulties in constructing a story around the illness may foster skepticism and disbelief in others that serve to delegitimate the illness. However, for their part, physicians may distrust the FMS label and the disease entity that it represents, but may nonetheless utilize the diagnosis as a means of managing the medical encounter. Whilst the physician has the formal authority to make the diagnosis, the patient can decide to resist this, such that the diagnosis is more a matter of reciprocal negotiation than of unilateral application. Medical expertise can thereby be acknowledged but also challenged.

\section{Methodological considerations}

The contribution of our metasynthesis depends on the quality of the primary studies and the conduct of the synthesis. Although the majority of primary studies fulfilled most of the methodological criteria, a number lacked information on issues relating to theoretical perspective and reflexivity as to how theory and tacit knowledge influenced interpretations. However, apparent methodological shortcomings are not a reason for automatically 
disregarding the insights of a study, ${ }^{72}$ and we found that both 'stronger' and 'weaker' studies contributed to developing our interpretation. Together, the included studies provided detailed and varied information. The importance of having rich data to inform a research question is in line with our interpretivist perspective. ${ }^{73}$

Confidence in the findings of a synthesis relies on the coherence of a particular finding with evidence in the primary studies, and the adequacy of such evidence. ${ }^{74}$ By maintaining the connection between the extracts of primary studies and the identified concepts during the analysis, we could support our interpretations with nuanced information in presenting our findings. In sum, we think that we have succeeded in developing themes across the studies supported by data as well as lines of arguments linking these themes together. We acknowledge, however, that a meta-ethnography will inevitably be partially a 'product of the synthesizer', ${ }^{24}$ p25 and ours should be interpreted in the light of this observation. Moreover, although the studies included were conducted in a number of countries, there appears to be considerable commonality in individuals' diagnostic experience in FMS. Nonetheless, future research might fruitfully explore possible cross-cultural aspects of this topic.

\section{Conclusion}

Through this metasynthesis, we have explored some core aspects of patients' diagnostic experience. The diagnosis is sought in the hope of making sense of the illness experience and providing both personal and social legitimation of an invisible and contested illness. However, as the individual's trajectory through the process of diagnosis unfolds, in many instances such hopes and expectations are disappointed, as the diagnosis does not resolve uncertainty, provides little in the way of lasting reassurance, does not lead to effective therapy, and fails to restore a sense of moral credibility. In the process, relationships with health professionals and within the person's social circle are often strained by trivialization, distrust, or explicit or implicit moral disfavour. In examining diagnosis in contested illness, and in FMS in particular, it is important to avoid what McGann ${ }^{8}$ calls the 'diagnostic imaginary.' By viewing diagnoses as 'morally neutral, scientifically valid, ontologically real "things" rather than sociopolitical achievements, ${ }^{8}$ p337 this way of thinking overlooks the complex social and political processes that underpin the diagnostic process.

\section{Acknowledgements}

We thank Senior Librarian Hilde L. Flaatten at the Medical Library, University of Oslo, Norway, for conducting the systematic literature search. 


\section{References}

1. Jutel A. Sociology of diagnosis: a preliminary review. Sociol Health Illn 2009; 31(2): 278-299.

2. Kleinman A. The illness narratives. Cambridge, MA: Basic Books, 1988.

3. Blaxter M. Diagnosis as category and process: The case of alcoholism. Soc Sci Med 1978; 12(1): 9-17.

4. Horwitz A. Creating mental illness. Chicago: University of Chicago Press, 2002.

5. Nettleton S. "I just want permission to be ill": towards a sociology of medically unexplained symptoms. Soc Sci Med 2006; 62(5): 1167-1178.

6. Brown P. Naming and framing: the social construction of diagnosis and illness. $J$ Health Soc Behav 1995; (Extra Issue): 34-52.

7. Wainwright D, Calnan M, O'Neil C, et al. When pain in the arm is "all in the head": The management of medically unexplained suffering in primary care. Health Risk Soc 2006; 8(1): 71-88.

8. McGann PJ and Hutson DJ (eds). Sociology of diagnosis. Bingley: Emerald, 2011.

9. Lemert E. Social pathology: A systematic approach to the theory of sociopathic behavior. New York: McGraw-Hill, 1951.

10. Strauss A. Negotiation: varieties, contexts, processes and social order. San Fransisco, CA: Jossey-Bass, 1976.

11. Gerhardt U. Ideas about illness. An intellectual and political history of medical sociology. New York, NY: New York University Press, 1989.

12. Conrad P and Barker KK. The social construction of illness: key insights and policy implications. J Health Soc Behav 2010; 51(suppl): 67-79.

13. Conrad P and Schneider JW. Deviance and medicalization. From badness to sickness. 2nd ed. Philadelphia, PA: Temple University Press, 1992.

14. Wolfe F, Smythe HA, Yunus MB, et al. The American College of Rheumatology 1990 Criteria for the Classification of Fibromyalgia. Report of the Multicenter Criteria Committee. Arthritis Rheum 1990; 33(2): 160-172.

15. Wang S-M, Han C, Lee S-J, et al. Fibromyalgia diagnosis: a review of the past, present and future. Expert Rev Neurother 2015; 15(6): 667-679.

16. Smythe HA. Referred pain and tender points. Am J Med 1986; 81(3A): 90-92.

17. Chinn P, Caldwell W, Gritsenko F. Fibromyalgia pathogensis and treatment options update. Curr Pain Headache Rep 2016; 20(4): 25.

18. Wolfe F, Clauw DJ, Fitzcharles MA, et al. Fibromyalgia criteria and severity scales for clinical and epidemiological studies: a modification of the ACR preliminary diagnostic criteria for fibromyalgia. J Rheumatol 2011; 38(6): 1113-1122.

19. Ehrlich GE. Pain is real; fibromyalgia isn't. J Rheumatol 2003; 30(8): 1666-1667.

20. Hadler NM and Greenhalgh S. Labeling woefulness: the social construction of fibromyalgia. Spine 2008; 10(1): 1-4.

21. Sim J and Madden S. Illness experience in fibromyalgia syndrome: a metasynthesis of qualitative studies. Soc Sci Med. 2008; 67(1): 57-67.

22. Hannes K and Lockwood C. Synthesizing qualitative research. Choosing the right approach. Chichester: Wiley-Blackwell, 2012.

23. Paterson BL, Thorne SE, Canam C, et al. Meta-study of qualitative health research. A practical guide to meta-analysis and meta-synthesis. Thousand Oaks,CA: Sage, 2001. 
24. Noblit GW and Hare RD. Meta-ethnography: Synthesizing qualitative studies. London: Sage, 1988.

25. Turner S. Sociological explanation as translation. New York: Cambridge University Press, 1980.

26. Diver C, Avis M and Gupta A. Quest, chaos and restitution: the illness narratives of individuals diagnosed with fibromyalgia. In: Richards R and Creek J (eds). Chronicity, care and complexity. Oxford: Interdisciplinary Press, 2013, 25-40.

27. Barker KK. The fibromyalgia story: medical authority and women's world of pain. Philadelphia, PA: Temple University Press, 2005.

28. Joanna Briggs Institute. Reviewers' manual - 2014 edition. Adelaide: University of Adelaide, 2014.

29. Briones-Vozmediano E, Vives-Cases C, Ronda-Pérez E, et al. Patients' and professionals' views on managing fibromyalgia. Pain Res Manag 2013; 18(1): 19-24.

30. Madden S and Sim J. Creating meaning in fibromyalgia syndrome. Soc Sci Med 2006; 63(11): 2962-2973.

31. Undeland $\mathrm{M}$ and Malterud $\mathrm{K}$. The fibromyalgia diagnosis: hardly helpful for the patients? A qualitative focus grop study. Scand J Prim Health Care 2007; 25(4): 250255.

32. Madden S and Sim J. Acquiring a diagnosis of fibromyalgia syndrome: the sociology of diagnosis. Soc Theor Health 2016; 14(1): 88-108.

33. Colmenares-Roa T, Huerta-Sil G, Infante-Castaneda C, et al. Doctor - patient relationship between individuals with fibromyalgia and rheumatologists in public and private health care in Mexico. Qual Health Res 2016; 26(12): 1674-1688.

34. Cooper, S and Gilbert, L. An exploratory study of the experience of fibromyalgia diagnosis in South Africa. Health (London) 2017; 21: 337-353.

35. Hellström O, Bullington J, Karlsson G, et al. A phenomenological study of fibromyalgia. Patient perspectives. Scand J Prim Health Care 1999; 17(1): 11-16.

36. Schaefer KM. Struggling to maintain balance: a study of women living with fibromyalgia. J Adv Nurs 1995; 21(1): 95-102.

37. Sallinen M, Kukkurainen ML, Peltokallio L, et al. Fatigue, worry, and fear - life events in the narratives of women with fibromyalgia. Health Care Women Int 2012; 33(5): 473-494.

38. McMahon L, Murray C, Sanderson S, et al. "Governed by the pain": narratives of fibromyalgia. Disabil Rehabil 2012; 34(16): 1358-1366.

39. Henriksson C. Living with continuous muscular pain - patient perspectives. Part 1. Encounters and consequences. Scand J Caring Sci 1995; 9(2): 67-76.

40. Raymond MC and Brown JB. Experience of fibromyalgia. Qualitative study. Can Fam Physician 2000; 46(May): 1100-1106.

41. Sturge-Jacobs M. The experience of living with fibromyalgia: confronting an invisible disability. Res Theor Nurs Pract 2002; 16(1): 19-31.

42. Wuytack F and Miller P. The lived experience of fibromyalgia in female patients, a phenomenological study. Chirop Man Ther 2011; 19(1): 22.

43. Durif-Bruckert C, Roux P and Rousset H. Medication and the patient-doctor relationship: a qualitative study with patients suffering from fibromyalgia. Health Expect 2014; 18(6): 2584-2594. 
44. Arnold LM, Crofford LJ, Mease PJ, et al. Patient perspectives on the impact of fibromyalgia. Patient Educ Couns 2008; 73(1): 114-120.

45. Hallberg LRM and Carlsson SG. Psychosocial vulnerability and maintaining forces related to fibromyalgia. Scand J Caring Sci 1998; 12(2): 95-103.

46. Schaefer KM. The lived experience of fibromyalgia in African American women. Holist Nurs Pract 2005; 19(1): 17-25.

47. Dennis NL, Larkin M and Derbyshire SWG. "A giant mess" - making sense of complexity in the accounts of people with fibromyalgia. Br J Health Psychol 2013; 18(4): 763-781.

48. Homma M, Yamazaki $\mathrm{Y}$, Ishikawa $\mathrm{H}$, et al. 'This really explains my case!': biographical reconstruction of Japanese people with fibromyalgia meeting peers. Health Scociol Rev 2016; 25(1): 62-77.

49. Lempp HK, Hatch SL, Carville SF, et al. Patients' experiences of living with and receiving treatment for fibromyalgia syndrome: a qualitative study. BMC Musculoskelet Disord 2009; 10: 124.

50. Mengshoel AM and Heggen K. Recovery from fibromyalgia - patients' own experiences. Disabil Rehabil 2004; 26(1): 46-53.

51. Söderberg S, Lundman B and Norberg A. Struggling for dignity: The meaning of women's experiences of living with fibromyalgia. Qual Health Res 2001; 9(5): 575587.

52. Van Gordon W, Shonin E and Griffiths MD. Meditation awareness training for individuals with fibromyalgia syndrome: an interpretive phenomenological analysis of participants' experiences. Mindfulness 2016; 7(2): 409-419.

53. Cunningham MM and Jillings C. Individuals' descriptions of living with fibromyalgia. Clin Nurs Res 2006; 15(4): 258-273.

54. Armentor JL. Living with a contested, stigmatized illness: experiences of managing relationships among women with fibromyalgia. Qual Health Res 2017; 27: 462-473.

55. Dumit J. Illnesses you have to fight to get. Facts as forces in uncertain, emergent illnesses. Soc Sci Med 2006; 62(3): 577-590.

56. Ware NC. Suffering and the social construction of illness: The delegitimation of illness experience in chronic fatigue syndrome. Med Anthropol Q 1992; 6(4): 347-361.

57. Mik-Meyer N and Obling AR. The negotiation of the sick role: general practioners' classification of patients with medically unexplained symptoms. Sociol Health Illn 2012; 34(7): 1025-1038.

58. Reid J, Ewan C and Lowy E. Pilgrimage of pain: The illness experiences of women with repetition strain injury and the search for credibility. Soc Sci Med 1991; 32(5): 601-612.

59. Snelgrove S and Liossi C. Living with chronic low back pain: a metasynthesis of qualitative research. Chronic Illn 2013; 9(4): 283-301.

60. Swoboda DA. Negotiating the diagnostic uncertainty of contested illnesses: physicians practices and paradigms. Health (London) 2008; 12(4): 453-478.

61. Aronowitz RA. Making sense of illness. Science, society, and disease. Cambridge: Cambridge University Press, 1998.

62. Radley A. Making sense of illness. The social psychology of health and disease. London: Sage, 2004

63. Parsons T. The social system. London: Routledge and Kegan Paul, 1951. 
64. Frank AW. Illness as a moral occasion: restoring agency to ill persons. Health (London) 1997; 1(2): 131-148.

65. Frank AW. From sick role to narrative subject: An analytic memoir. Health (London) 2016; 20(1): 9-21.

66. Zavestoski S, Brown P, McCormick S, et al. Patient activism and the struggle for a diagnosis: Gulf War illnesses and other medically unexplained physical symptoms in the US. Soc Sci Med 2004; 58(1): 161-175.

67. Grape HE, Solbrække KN, Kirkevold M, et al. Staying healthy from fibromyalgia is ongoing hard work. Qual Health Res 2015; 25(5): 679-688.

68. Bülow P. "You have to ask a little": troublesome storytelling about contested illness. In: Hydén L-C and Brockmeier J (eds). Health, illness and culture. Broken narratives. New York: Routledge, 2008: 131-53.

69. Williams G. The genesis of chronic illness: narrative re-construction. Sociol Health Illn 1984; 6(2): 175-200.

70. Sools A and Murray M. Promoting health through narrative practice. In: Murray M (ed). Critical Health Psychology. 2nd ed.Houndmills: Palgrave Macmillian, 2015: 235-53.

71. Toye F, Seers K, Allock N, et al. Patients' experiences of chronic non-malignant musculoskeletal pain: a qualitative systematic review. Br J Gen Pract 2013; 63(617):e829-e841.

72. Pawson R. Digging for nuggets: How 'bad' research can yield 'good' evidence. Int $J$ Soc Res Methodol 2006; 9(2):127-142.

73. Cohen DJ, Crabtree BF. Evaluative criteria for qualitative research in health care: Controversies and recommendations. Ann Fam Med 2008; 6(4):331-339.

74. Lewin S, Glenton C, Munthe-Kaas H, et al. Using qualitative evidence in decision making for health and social interventions: An approach to assess confidence in findings from qualitative synthesis (GRADE-CerQual). PLOS Med 2015; 12(10): e1001895 